\title{
MATHEMATICAL MODEL OF LIQUIDS MIXTURE HYDRAULIC BLOW ACTION IN PIPE LINE
}

Nishonov Fayzullo Xolmirzayevich

Tashkent institute of architecture and civil engineering, 100011 Tashkent Uzbekistan. A. Navoiy 13 n.fayz_1988@mail.ru

Orcid ID 0000-0002-1218-018X

\section{Crossref}

\section{ABSTRACT}

The Mathematical model action hydraulic blow mixture liquids interval happens to in pipeline and moment of the action of the hydraulic blow. It Is Researched time and begin actions of the hydraulic blow mixture to liquids pipe line engineering complex in dependencies of the initial pressure, from length of the pipe and corner of the slopping of the pipe line

Keywords: mixtures of liquids, pulsation, biphasic liquids of the first and second liquid phases 
In orderly working pressure sluice (penstock), on which water evenly moves,

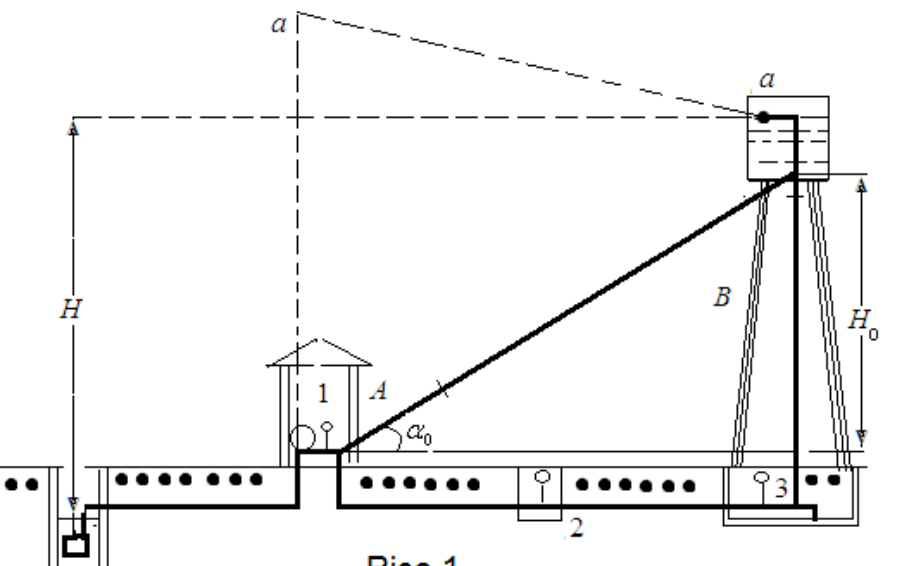

Rice.1. internal pressure, pumps or some other pressure devices (elevated source, reservoir, and water tower) originate i.e. pressure. Moreover, maximum mark of piezometric level is found in point of the location of device, creating pressure as defined on fig. 1. Pumping station $A$ is shown on this figure, giving water on horizontally laid pipe line in water tower $B$; pressures in pipe line are characterized by piezometric line $a-a$ manometers 1,2 and 3 are located along water pressure line. If by tail gate in water tower under normal work of giving pump quickly stop output of water in tank, that pressure in pipe line beside towers will sharply increase and this increase of the pressure is quickly passed along pipe line on direction to pumping station that can be observe on manometer 3,2 and 1. But if under normal work of whole device pumps will suddenly stop or will powerfully reduce water delivery, then pressure in pipe line will sharply decrease and this reduction of the pressure will be quickly passed on direction to water tower and will be noted in manometer 1,2 and 3. In some cases depending on working pressure in pipe line, his(its) sizes, velocities of the motion of water and elastic characteristic of water and pipe line material, this reduction of pressures can be so significantly that pressure inwardly of pipe line becomes the equal to atmospheric pressure. [1,3].

The hydraulic blow appears in general because of change the velocities of water delivery, it means that any reason, causing change of velocitiy, will

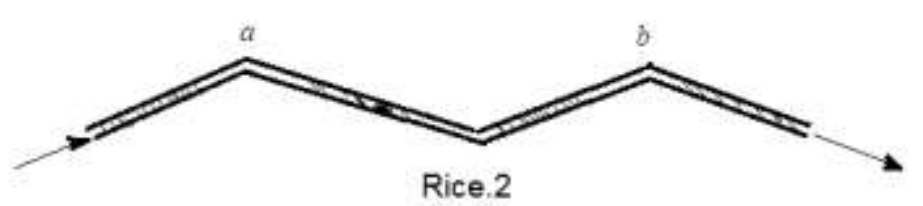
simultaneously generate the phenomena of the hydraulic blow. In practice it is noticed that presence of the air in pipe line causes the blows and are explained by condition of the accumulation of the air in pipe line, which usually is accumulating in raised point of the pipe line (pic.2.), forming in them airbags, through which given water can break only by way of pulsation, i.e. with variable speed. On the other hand, at 
presence of the air accumulation in raised point $a$ and $b$ of the pressure line it is appears elastically nipped from two sides column of water, which can come to oscillatory motion; will cause dangerously high pressures and significant losses of the work if and when period of the own fluctuations of the column of water $a-b$ will be equal or divisible to duration of the turn of the pump, since then appears the phenomena of the resonance and as result significant increasing pressures. [2,5,6]

As it was shown above, at opening of pipeline valve motion of liquids will be transient and Bernoulli equation, with consideration of pressure loss on length and inertial term, will be written as:

$$
\frac{p_{1}}{\rho}+\frac{V_{1}^{2}}{2 g}+z(t)=\frac{p_{0}}{\rho}+\frac{\vartheta_{2}^{2}}{2 g}+\lambda \frac{z(t)}{d} \frac{\vartheta^{2}}{2 g}+z(t) \frac{j}{g}
$$

where: $j=\frac{d \vartheta}{d t}$ accelerative pressure. From equality (1) shall get the following equation for average velocity of given water $\vartheta(t)$ in inclined pipe line;

$$
\frac{d \vartheta}{d t}=g \sin \alpha-\frac{\lambda}{2 d_{0}} \vartheta^{2}
$$

At the moment of pipeline valve close the pressure in pipe line beside water tower will sharply increase and this increasing of the pressure is quickly transmitted along pipe line on direction to pumping station and appears hydraulic blow, for which it is a following initial conditions, under $t=0$, velocity of water inwardly pipe line $\vartheta=0$, then equation to average velocity of water motion in pipe line

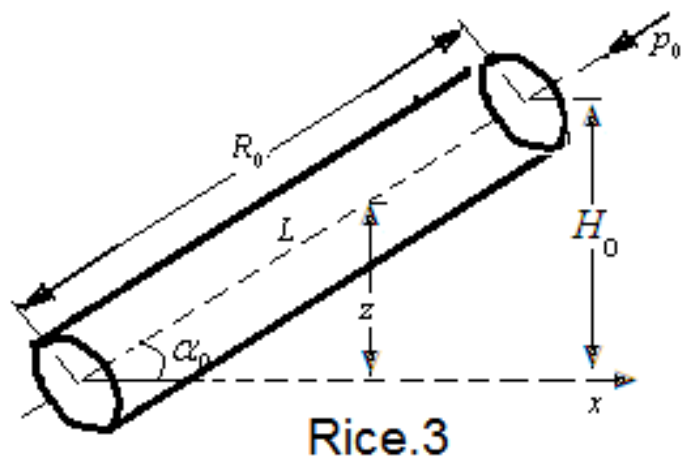

(2) reduces to common differential equation $\frac{d \vartheta}{a_{0}^{2}-\vartheta^{2}}=k d t$

Decision, which is found as integration will be:

$$
k=\frac{\lambda}{2 a_{0}} \text { Where: } a_{0}=\sqrt{\frac{g \sin \alpha}{k}}
$$

In addition, vertical component of the vector to velocities of the liquids' moving particles is defined by equality:

$$
\frac{d z}{d t}=-\frac{\vartheta(t)}{\sin \alpha}
$$


Whence we find the equation of the moving the front of the water wave in considered part a pipeline:

$$
\frac{d z}{d t}=a_{1} \frac{e^{2 a_{1} k\left(t-t_{0}\right)}-1}{e^{2 a_{1} k\left(t-t_{0}\right)}+1}
$$

Where:

$$
a_{1}=a_{0} \sin \alpha
$$

Now we research the time of the full filling of water tower $B$ (pic.1). Then initial condition for determination of change the height of the filling tower $B$ will:

$$
z(t)=L_{0} \sin \alpha, \quad z(t)=-\frac{1}{k} \ln \left(1+\exp \left(2 a_{0} k t\right)\right)+C
$$

Considering, initial condition we shall get law of the change of the back front of the wave of the water column $z(t)$ which is of the form of:

$$
z(t)=\sqrt{\frac{g}{k}} t-\ln \frac{(1+\exp (2 \sqrt{g k} t)}{2}+H_{0}
$$

We shall expect that under $t=T$ inclined pipe line completely is filled, then will exist the equality $z(T)=0$. Whence considering equality (3) we shall get the equation:

$$
\sqrt{g k} T-k H_{0}=\ln \left[\frac{1+\exp (2 \sqrt{g k} T)}{2}\right]
$$

Getting exponentiation, we find the expression for full filling the water tower:

$$
2 \exp (\sqrt{g k} T) \exp \left(k H_{0}\right)=1+\exp (2 \sqrt{g k} T)
$$

Entering indications, $F(T)=\exp (\sqrt{g k} T)$ we shall get the quadratic equation for $F(T)$ function:

$$
F^{2}(T)-2 \exp k H_{0} F(T)+1=0
$$

Considering, feature of function $F(T)$, that $F(T)>1$ the decision of equation we shall write in the manner of:

$$
F(T)=\exp \left(k H_{0}\right)+\sqrt{\exp \left(2 k H_{0}\right)-1}
$$

Finding the logarithm of expression, we find time of the full filling to water tower $B$

$$
T=\frac{1}{\sqrt{g k}} \ln \left(\exp \left(k H_{0}\right)+\sqrt{\exp \left(2 k H_{0}\right)-1}\right)
$$

At lack of the pressure loss during a friction on length of the pipe line i.e. under $\lambda \rightarrow 0$ we shall have an expression:

$$
T_{0}=\lim _{\lambda \rightarrow 0} T=\lim _{\lambda \rightarrow 0} \frac{1}{g k}\left[\exp \left(k H_{0}\right)+\exp g 2 k H_{0}-1\right]
$$


Full filling of water tower during action of the hydraulic blow in dependence of the initial pressure, from length and inclination corner of the pipe line is of the form of:

$$
T_{0}=\sqrt{\frac{2 H_{0}}{g \sin \alpha}}=\sqrt{\frac{2 L_{0}}{g}},
$$

2. For research of given problems for mixture of the two-phase liquid, we write Bernoulli as in work $[4,7]$ i.e.:

$$
\begin{aligned}
& p_{0}+\frac{1}{2} \rho_{1 i} \Lambda \vartheta_{c M}^{2}+\rho_{2 i}\left(f_{1}+f_{2} \frac{\rho_{2 i}}{\rho_{1 i}}\right) g z= \\
& =p_{0}+\frac{1}{2} \rho_{1 i} \Lambda \vartheta_{c M}^{2}+\lambda_{c M} \frac{z}{d} \rho_{1 i} \Lambda \vartheta_{c M}^{2}+z \rho_{1 i}\left(f_{1}+f_{2} \frac{\rho_{2 i}}{\rho_{1 i}}\right) \frac{d \vartheta_{c M}}{d t} \\
& \quad \Lambda=\left(f_{1}+f_{2} \frac{\rho}{\rho-1}\right)^{2}\left[\frac{1-(1-\rho) f_{1}}{f_{1}\left(1-f_{1}\right)} j\right], \rho=\frac{\rho_{2 i}}{\rho_{1 i}}
\end{aligned}
$$

Where:

$\rho_{1}, \rho_{2}$-density, $\vartheta_{1}, \vartheta_{2}$ - a velocities accordingly of first and the second phase of liquids.

After adduction of the terms containing factor of concentrations of the mixture phases, Bernoulli equation comparatively to mixture is of the form of:

$$
\begin{aligned}
& \rho_{1 i}\left(f_{1}+f_{2} \rho\right) g z=\lambda_{c M} \frac{z}{d} \rho_{1 i} \Lambda \vartheta_{c M}^{2}+ \\
& +z \rho_{1 i}\left(f_{1}+f_{2} \rho\right) \frac{d \vartheta_{c M}}{d t} \frac{1}{g} \frac{d \vartheta_{a}}{d t}+\frac{\lambda_{c M}}{2 g d} \vartheta_{c M}^{2}
\end{aligned}
$$

Considering that, small factors of power of pipe walls resistance has:

$$
\left(f_{1}+f_{2} \rho\right) \frac{d \vartheta_{c u}}{d t}=\left(f_{1}+f_{2} \rho\right) g-\lambda_{c u} \frac{\Lambda}{d} \vartheta_{c u}^{2}
$$

Thence we find change of moving mixture velocities at time:

$$
\frac{d \vartheta_{c u}}{d t}=g-\frac{\lambda_{c u} \Lambda}{d\left(f_{1}+f_{2} \rho\right)} \vartheta_{c u}^{2}
$$

Entering following indications:

$$
\frac{d \vartheta_{c u}}{g d t}=\frac{d \vartheta}{d \tau}, \quad \vartheta_{c u}=\vartheta \sqrt{H_{0} g}, \quad t=\sqrt{\frac{H_{0}}{g}} \tau
$$

From Bernoulli equation (5) we find expression for length of the hydraulic blow, which we shall identify through $\tau$ :

$$
\sqrt{H_{0} g} \sqrt{\frac{g}{H_{0}}} \frac{d \hat{\vartheta}}{d \tau}=g-\frac{\lambda_{c u} \Lambda H_{0} g}{d\left(f_{1}+f_{2} \hat{\rho}\right)} \hat{\vartheta}_{c u}^{2}
$$


Solving equation comparatively to time of the action of the hydraulic blow (closing the pipe valve), have a common differential equation:

$$
\frac{d \vartheta_{c u}}{d \tau}=1-\lambda_{c m} \frac{\Lambda H_{0}}{d\left(f_{1}+f_{2} \rho\right)} \vartheta_{c m}^{2}
$$

The decision of the differential equation is of the form of:

$$
d \vartheta=+A \frac{\exp \left(\frac{2 \tau}{A}\right)-1}{\exp \left(\frac{2 \tau}{A}+1\right)} d \tau=+A d \tau+2 A
$$

By differentiating of this expression, we find the velocity at moment of the hydraulic blow:

$$
\vartheta=+A \tau+A^{2} \int \frac{1}{\exp \frac{2 \tau}{A}+1} d \tau .
$$

By integrating (7) we find:

$$
\vartheta+1=+A \tau+A^{2} \ln \left(\frac{1+\exp \left(\frac{2 \tau}{A}\right)}{2}\right)
$$

By exponentiation of expressions, we find the formula for velocity of liquids mixture in pipeline:

$$
\vartheta=1+A \tau+A^{2}\left(1+\exp \frac{2 \tau}{A}\right)-A^{2} \ln 2
$$

As it is well known, velocity at moment of the hydraulic blow is a zero so from equality (8) we have:

$$
\begin{gathered}
\vartheta=1-A \tau+A^{2} \frac{2 \tau}{A}-A^{2} \ln 2=0, \\
1+A \tau=A^{2} \ln 2 ; \quad \tau=\frac{A^{2} \ln 2-1}{A}, \vartheta=A \tau+l, \vartheta=A \tau+l-\ln \frac{1+\exp \frac{2 \tau}{A}}{2}
\end{gathered}
$$

By exponentiation, this expression shall have:

$$
\exp [A \tau+l-z]=\frac{1}{2}\left(1+\exp \frac{2 \tau}{A}\right)
$$

Under $t=T$ from expressions (8) we have:

$$
\tau=T \sqrt{\frac{g}{H_{0}}}, z(\tau)=0 .
$$

Then from expression (8) we shall get the quadratic equations: 


$$
2 \exp [A \tau+2 l]=1+\exp \frac{2 \tau}{A}
$$

Quadratic equation (9) we write comparatively for term $\exp \frac{2 \tau}{A}$ whence we have:

$$
\exp \frac{2 \tau}{A}-2 \exp A \tau \exp 2 l+1=0
$$

Solving this equation comparatively to space of time of the hydraulic blow, we find:

$$
\exp \frac{\tau_{T}}{A}=\exp (2 l) \pm \sqrt{\exp (2 l)-1}
$$

since that, $\exp \left(\frac{\tau_{T}}{A}\right)>1$ we find the expression for moment of the hydraulic blow,

$$
\tau_{T}=A \ln \left[e^{1 \hat{\imath}}+\sqrt{\exp 2 \hat{l}-1}\right]
$$

At lack of friction, time for full filling the tower $B$ is defined by equality:

$$
T=\sqrt{\frac{2 H_{0}}{g}} \Lambda_{0}
$$

Wave of reduction of the pressure will get to tower $B$ during 4,32 second and will cause inverse wave of increasing of the pressure the same power, which, moving forward to pumping station $A$ will restore statistical pressure on passed area of water conduit. Through 4,32 second reflected wave will get to pumping station and will meet locked inverse valve, from which it is reflected with same sign, and in pipe line it will appear wave of increased pressure with power $+140,3$ м.вод.cm., which from station $A$ moves to tower $B$ If average velocity of the water motion in pipe line $\vartheta_{c m}=1,17 \frac{\mathrm{m}}{\mathrm{sek}}$ and average velocity of the spreading the shock wave $a_{0}=1157 \frac{\mathrm{m}}{\mathrm{sek}}$ then according formula (3) and (7) we find corresponding value on lower area of water conduit, adjoining to station $A$ which are: $\vartheta_{c m 1}=1,10 \frac{\mathrm{m}}{\mathrm{sek}}$ И $a_{1}=1143 \frac{\mathrm{m}}{\mathrm{sek}}$. 


\section{References}

1. Bernar Le Meote. "Introduction to hydrodynamics and theory of the waves on water". Gidrometeoizdat. Leningrad-1974g.368s.

2. Kiselev P.G. The Hydraulics and bases mechanical engineers to liquids. Moscow "Energy" 1980. $360 \mathrm{~s}$.

3. Surin A.A. The Hydraulic blow in pipe line and fight with him. Moscow. 1990г. 386c.

4. $\quad$ Nigmatulin R.I. The Track record poliphase sred.- M.: Science, 1987. $464 \mathrm{~s}$. 5. Shashin V.M. "Gidromehanika" Moscow "High school" 1990г.385c.

6. Hamidov A.A., Hudaykulov S.I., Mahmudov I.E. "Gidromehanika" FAN2008G. 340c.

7. Hamidov A.A., Hudaykulov S.I. The Theory stream poliphase viscous liquids. Tashkent- 2003."FAN". 